\title{
LARGE-ANGLE OSCILLATIONS IN HEAVY ION ELASTIC AND INELASTIC SCATTERING
}

\author{
R. DA SILVEIRA \\ Institut de Physique Nucléaire, Division de Physique Théorique $\left(^{*}\right)$ \\ 91406 Orsay, France \\ and \\ Ch. LECLERQ-WILLAIN $\left({ }^{* *}\right)$ \\ Université Libre de Bruxelles, Physique Nucléaire Théorique \\ 1050-Bruxelles, Belgique
}

(Reçu le 24 janvier 1975, accepté le 4 mars 1975)

\begin{abstract}
Résumé. - Le comportement oscillatoire observé aux grands angles dans les sections efficaces de diffusion élastique et inélastique d'ions lourds aux énergies supérieures à la barrière de Coulomb, est interprété comme étant dû à un effet d'interférence entre l'amplitude de diffusion dispersive arc en ciel et celle provenant de la branche négative de la fonction de déflexion classique.
\end{abstract}

\begin{abstract}
The oscillatory behaviour observed at large angles in heavy ion elastic and inelastic scattering cross-sections at energies above the Coulomb barrier, is interpreted as an interference effect between rainbow scattering and the contribution coming from the negative branch of the classical deflection function.
\end{abstract}

In heavy ion reactions, at energies near and above the Coulomb barrier, the structure of the elastic and inelastic cross-sections, strongly 'depends on the scattering angle region. On one side of the grazing angle $\theta_{\mathrm{r}},\left(\theta<\theta_{\mathrm{r}}\right)$ the elastic and inelastic crosssections present an out of phase oscillatory structure whatever be the parity of the angular momentum transfered $[1,2]$. This oscillatory behaviour has already been interpreted as a quantal interference effect between two classical trajectories which contribute to the elastic and inelastic cross-sections at each scattering angle [3, 4].

On the other side of $\theta_{\mathrm{r}},\left(\theta>\theta_{\mathrm{r}}\right)$ the elastic and inelastic cross-sections drops rapidly to very low values. However, in a recent experiment [5] it appears that in place of this common monotonic decrease, we observe well defined in phase oscillations (see figure 1 and reference [5]).

The aim of the present letter is to show how this oscillatory behaviour can be qualitatively [6] and quantitatively explained in terms of semi-classical arguments.

The contribution of the classical deflection function $\theta(l)$ to the semi-classical scattering amplitude

(*) Laboratoire associé au C.N.R.S.

$\left({ }^{* *}\right)$ Maître de Recherches au F.N.R.S. starts at the $l$ value $\left(l_{\mathrm{a}}\right)$ for which $\theta(l)=-\infty$ [7]. For $l \leqslant l_{\mathrm{a}}$, all partial waves are completly absorbed. For $l>l_{\mathrm{a}}, \theta(l)$ has the shape shown in the upper part of figure 1 ; one negative branch and two positive ones. The angle $\theta_{\mathrm{r}}$ is defined by $\theta^{\prime}(l)_{l=l_{\mathrm{r}}}=0$.

As long as the incident energy remains slightly above the Coulomb barrier, the negative branch has a negligible contribution to the total scattering amplitude.

For $\theta>\theta_{\mathrm{r}}$ the contribution of the positive branches, when evaluated in the simplest approximation (the Airy approximation [8]) gives the above mentioned decreasing behaviour or rainbow effect (dotted curve, Fig. 1). When the energy increases, the contribution of the negative branch is no longer negligible, particulary for angles where the rainbow scattering crosssections has fallen to very low values, i.e. for $\theta \gg \theta_{\mathrm{r}}$.

Taking into account the negative branch with $l$ values denoted by $l_{\mathrm{n}}$, the semi-classical elastic crosssection is defined by [8]

$$
\frac{\mathrm{d} \sigma}{\mathrm{d} \Omega}=\sigma_{\mathrm{r}}(\theta)+\sigma_{\mathrm{n}}(\theta)-2\left(\sigma_{\mathrm{r}} \sigma_{\mathrm{n}}\right)^{1 / 2} \cos \left(\delta_{\mathrm{r}}-\delta_{\mathrm{n}}\right)
$$

where

$$
\sigma_{\mathrm{n}}(\theta)=\frac{l_{\mathrm{n}}}{k^{2} \sin \theta\left|\theta^{\prime}(l)\right|_{l_{\mathrm{n}}}}
$$




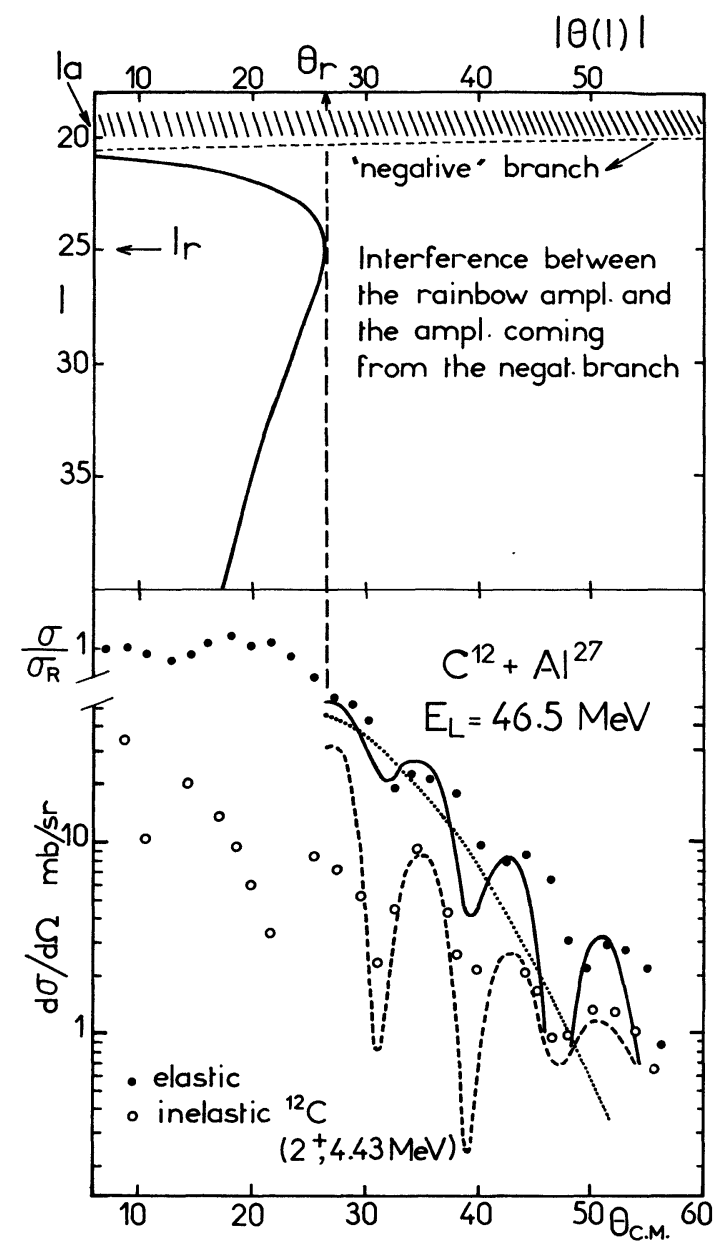

FIG. 1. - Elastic and inelastic scattering cross-sections of $\mathrm{C}^{12}$ incident on $\mathrm{Al}^{27}$ at $E_{\mathrm{L}}=46.5 \mathrm{MeV}$. Upper part : classical deflection function. In order to keep reasonable proportions in this figure we have reflected the negative branch (broken curve) with respect to the $l$-axis. Lower part : comparison of the experimental data with the theoretical predictions. Elastic cross-section evaluated with (full curve) and without (dotted curve) the contribution of the negative branch. Broken curve, inelastic cross-section.

and $\quad \sigma_{\mathrm{r}}(\theta)=\frac{2 \pi l_{\mathrm{r}}}{k^{2} \sin \theta q^{2 / 3}} \mathrm{Ai}^{2}\left[\left(\theta-\theta_{\mathrm{r}}\right) / q^{1 / 3}\right]$

are respectively, the classical cross-section for the negative branch and the Airy approximation [8] for the rainbow scattering (the contribution from the two positive branches); $q$ is the curvature of $\theta(l)$ at $l=l_{\mathrm{r}}$. In (1) the phase factors $\delta_{\mathrm{r}}$ and $\delta_{\mathrm{n}}$ can be expressed in terms of the W.K.B. phase shifts $\eta\left(l_{\mathrm{r}}\right)$ and $\eta\left(l_{\mathrm{n}}\right)$ by

and

$$
\begin{gathered}
\delta_{\mathrm{r}}=2 \eta\left(l_{\mathrm{r}}\right)-\theta l_{\mathrm{r}}-\pi / 4 \\
\delta_{\mathrm{n}}=2 \eta\left(l_{\mathrm{n}}\right)+\theta l_{\mathrm{n}}+\pi / 2 .
\end{gathered}
$$

In the region $\theta>\theta_{\mathrm{r}}, \sigma_{\mathrm{r}}(\theta)$ decreases more rapidly than $\sigma_{\mathrm{n}}(\theta)$ and the interference term in (1) produces oscillations whose amplitudes becomes more pronounced with increasing $\theta$. The wave length of these oscillations, obtained from (4), is $\Delta \theta=2 \pi /\left(l_{\mathrm{r}}+l_{\mathrm{n}}\right)$, i.e. almost independent of $\theta$.

Now we shall analyse the inelastic scattering cross-section. To do this, we take the semi-classical limit of the D.W.B.A. expression for the reaction amplitude (for details see reference [4] and [9]). The result we obtain is

$$
\begin{aligned}
\frac{\mathrm{d} \sigma}{\mathrm{d} \Omega_{0 \rightarrow I}} \sim \sum_{\mu} \mid & \left.Y_{I \mu}\left(\frac{\pi}{2}, 0\right)\right|^{2}\left\{\sigma_{\mathrm{r}}(\theta)\left|a_{\mathrm{r}}\left(l_{\mathrm{r}}, I, \mu\right)\right|^{2}+\right. \\
& +\sigma_{\mathrm{n}}(\theta)\left|a_{\mathrm{n}}\left(l_{\mathrm{n}}, I, \mu\right)\right|^{2}-2(-)^{I}\left(\sigma_{\mathrm{r}} \sigma_{\mathrm{n}}\right)^{1 / 2} \\
& \left.\times\left|a_{\mathrm{r}}\right|\left|a_{\mathrm{n}}\right| \cos \left(\delta_{\mathrm{r}}-\delta_{\mathrm{n}}\right)\right\}
\end{aligned}
$$

where $a_{\mathrm{r}, \mathrm{n}}\left(l_{\mathrm{r}, \mathrm{n}}, I, \mu\right)$ are the inelastic amplitudes evaluated for $l=l_{\mathrm{r}}$ and $l-l_{\mathrm{n}}$ respectively.

From expression (5) and from its comparison with (1) we obtain two important conclusions :

i) The oscillations in the inelastic cross-section of even and odd parities, are out of phase [10].

ii) The oscillations in the inelastic cross-section should be in phase or out of phase with the elastic oscillations, according to whether the parity of the excited state is even or odd. This phase rule between the elastic and inelastic cross-section is just the inverse of the well known Blair phase rule [11] $\left({ }^{1}\right)$.

The results of a numerical calculation with expressions (1) and (5) for the elastic scattering and inelastic $2^{+}(4.43 \mathrm{MeV})$ excitation of $\mathrm{C}^{12}$ incident on $\mathrm{Al}^{27}$ at $E_{\mathrm{L}}=46.5 \mathrm{MeV}$ [5] are presented in figure 1. The classical deflection function (upper part of Fig. 1) has been calculated with a Coulomb plus WoodsSaxon nuclear potential, with the parameters $V_{0}=35 \mathrm{MeV}, r_{0}=1.15 \mathrm{fm}$ and $a=0.55 \mathrm{fm}$. The inelastic results are obtained in absolute scale with a deformation parameter $\beta_{2}=0.3$ for the $2^{+}$state in $\mathrm{C}^{12}$.

In spite of the crude approximation used in the description of the rainbow scattering, the essential features of the experimental results are quite well reproduced.

(1) The Blair phase rule is applicable at higher energies where the Coulomb amplitude is less important.

\section{References}

[1] Christensen, P. R., Chernov, I., Gross, E. E., Stokstad, R. and Videback, F., Nucl. Phys. A 207 (1973) 433.

[2] FoRD, J. L. C. Jr, Toth, K. S., Hensley, D. C., GaEdKe, R. M., Riley, P. J. and Thornton, S. T., Phys. Rev. C 8 (1973) 1912.
[3] Malfliet, R. A., Landowne, S. and Rostokin, V., Phys. Lett. 44B (1973) 238;

Broglia, R. A., Landowne, S., Malfliet, R. A., Rostokin, V. and Winther, A. A., Phys. Report 11C (1974) 1.

[4] Da Silveira, R. and Leclerq-Willain, Ch., Orsay Rep. IPNO/TH 73-52. 
[5] Pougheon, F., Detraz, C., Rotbard, G. and Roussel, P., Proc. of the Int. Conf. on React. Betw. Compl. Nuclei (Nashville) 1974, suppl. vol. I, p. 4.

[6] Da Silveira, R., LeclerQ-Willain, Ch. and Pougheon, F., Same as for reference 5, p. 5 .

[7] Da Silveira, R., Phys. Lett. 45B (1973) 211 and in Proc. of the Int. Conf. on Nucl. Phys. (Munich) 1973 contribution No 540 .

[8] Ford, K. W. and Wheeler, J. A., Ann. Phys. 7 (1959) 259.

[9] Da Silveira, R. and LeclerQ-Willain, Ch., to be published.

[10] See also Knoll, J. and SchaEFFER, R., Saclay Rep. DPhT/74. 